\title{
Transient Elastographic Values of Healthy Volunteers in a Tertiary care Hospital
}

\author{
Bhupendra Kumar Basnet, ${ }^{1}$ Sudhamshu KC, ${ }^{1}$ Dilip Sharma, ${ }^{1}$ Dinesh Shrestha ${ }^{1}$ \\ 'Department of Medicine, National Academy of Medical Sciences, Bir Hospital, Kathmandu, Nepal.
}

\section{ABSTRACT}

Introduction: Transient elastography is a very promising noninvasive procedure to determine liver stiffness for diagnosis of fibrosis in various chronic liver diseases. However, studies on normal values of liver stiffness in apparently healthy subjects are still few. We aimed to determine liver stiffness values in healthy Nepalese volunteers.

Methods: Transient elastography (Fibro ScanR, Echosens, Paris, France) was performed to find out liver stiffness values in 45 apparently healthy volunteers after explaining study protocol. Complete medical examination with routine laboratory tests was performed. Subjects with normal liver biochemistries and normal liver ultrasonography were taken for analysis.

Results: Mean liver stiffness value of study subjects was $4.24 \pm 0.70 \mathrm{kPa}$. Liver stiffness value was found higher in males than in females $(4.32 \pm 0.74 \mathrm{vs} 4.07 \pm 0.61 \mathrm{kPa}$, respectively, $\mathrm{P}=0.26)$ but not statistically significant. Similarly, comparison between age and liver stiffness also showed positive correlation $(r=0.211)$ but not statistically significant $(\mathrm{P}=0.164)$

Conclusions: Our study showed that the mean liver stiffness value was $4.24 \pm 0.70 \mathrm{kPa}$ in our population and influence of age, gender and body mass index were not significant.

Keywords: chronic liver disease; Fibro ScanR; healthy volunteers; liver stiffness valve; transient

elastography.

\section{INTRODUCTION}

Chronic liver diseases often result in fibrosis that may eventually lead to cirrhosis, a state that carries a risk of lethal complications, including hepatocellular carcinoma. ${ }^{1,2}$ The prognosis and management of chronic liver diseases often depend strongly on the degree of liver fibrosis. ${ }^{3}$

These facts point out the clinical interest in quantifying hepatic fibrosis and detecting patients with cirrhosis. At present, liver biopsy is the "gold standard" method to assess the grade of liver fibrosis. However, the use of liver biopsy has several limitations: physical and mental discomfort of the patients that may lead to a high percent of refusal, non-negligible morbidity and occasional mortality. ${ }^{4-6}$ Liver biopsy examination is difficult to repeat in generally asymptomatic patients. Furthermore, due to the limited size of liver samples and the subjective assessment made by pathologists, accuracy and reproducibility of histological grading has been questioned. Therefore, there is an increasing need for alternative noninvasive methods to estimate

Correspondence: Dr. Bhupendra Kumar Basnet, Department of Medicine, National Academy of Medical Sciences, Bir Hospital, Kathmandu, Nepal. Email: bhupendrakumarbasnet@gmail.com, Phone: $+977-9851059400$ 
the grade of liver fibrosis. ${ }^{7}$ Among the other potentially interesting approaches, elastography seems to be one of the most promising. Indeed, it is well known that liver stiffness is related to the degree of hepatic fibrosis, and palpation has been used from decades to establish a clinical diagnosis of hepatic fibrosis and cirrhosis.

Transient elastography (FibroScanR, Echosens, and Paris, France) is a recently described non-invasive procedure which consists of a $5-\mathrm{MHz}$ ultrasound transducer probe mounted on the axis of a vibrator. The vibrator generates a completely painless vibration (with a frequency of $50 \mathrm{~Hz}$ and amplitude of $2 \mathrm{~mm}$ ) which generates an elastic share wave propagating through the skin and the subcutaneous tissue to the liver. The velocity of the wave is directly related to the tissue stiffness which allows evaluating liver stiffness. ${ }^{8}$ The basic principle of transient elastography is that the propagation velocity of a wave through a homogenous tissue is proportional to its elasticity, which is correlated with the amount of fibrosis in the liver. The FibroscanR test has the advantage of being pain-free, easy-to-learn and fast $^{9}$ and give result immediately.

In the context of growing burden of chronic liver disease in our country, we need a novel, noninvasive and bedside method like transient elastography to assess liver fibrosis by measuring liver stiffness. Unlike liver biopsy, transient elastography can be repeated without risk to patients. Since, transient elastography is a relatively new diagnostic tool and it has been tested in different chronic liver disease but there are few data regarding healthy population. There are no published studies of transient elastography on either healthy or people with chronic liver diseases in Nepalese population. So, we aimed in this study to see the transient elastographic values in apparently healthy Nepalese population.

\section{METHODS}

This was a cross sectional descriptive study done in Liver Unit, National Academy of Medical Sciences, Bir Hospital on July-August 2013.

Healthy volunteers were taken for study after explaining about study purpose and methods. Volunteers were Resident Doctors and Nursing Staffs working in Bir Hospial at the study time. Healthy Volunteers aged 18 years and above were included and those volunteers who had any liver disease or suspected of having systemic disease affecting liver like raised transaminases, fatty liver in ultrasonography and those not willing to participate in study were excluded. All volunteers gave informed consent and the study protocol was approved by the institutional review board (IRB), National Academy of Medical Sciences. Those who agreed to participate had a complete medical examination with laboratory tests on the same day. Data were collected for each volunteer: age, gender, ethnicity, occupation and vital signs. Similarly, history of liver diseases, diabetes, arterial hypertension, smoking and alcohol use were also recorded. Routine laboratory tests including complete haemogram, random blood glucose, renal function test, liver biochemistries, serum total protein, albumin and total cholesterol, serological tests for hepatitis B\&C virus was also done. Likewise, ulrasonography of the abdomen done where size of liver and spleen was recorded and chest $x$ - ray also carried out. Body mass index was calculated and classified according south Asian BMI value (Normal BMI: 18.0$22.9 \mathrm{~kg} / \mathrm{m}^{2}$, Overweight: $23.0-24.9 \mathrm{~kg} / \mathrm{m}^{2}$, Obesity: $>25 \mathrm{~kg} / \mathrm{m} 2) .{ }^{10}$

Then FibroscanR of all volunteers were done in Centre for Liver Disease next day by the same clinician who was blinded to clinical and biochemical profiles. FibroscanR was performed on the right lobe of the liver, through intercostals spaces, with the patient lying in dorsal decubitus with the right arm in maximal abduction. The operator placed the fibroscanR transducer perpendicularly to the intercostals space, assisted by time motion ultrasound images, located the probe in a liver portion at least $6 \mathrm{~cm}$ thick and free of large vascular structures and gallbladders and presses the probe button to commence the measurement. Ten valid measurements were performed to examine a patient with transient elastography. The median value of the ten valid measurements was considered representative of liver elasticity. The success rate was calculated as the number of valid measurements divided by the total number of measurements. Examinations with a success rate higher than $60 \%$ and interquartile range (IQR), which represents the intrinsic variability of $T E$, $<30 \%$ of the median were considered reliable. The results were immediately obtained after performance of TE and expressed as the median value of 10 validated measurements in kilopascals $(\mathrm{kPa})$, values ranging from 2.5 to $75 \mathrm{kPa}$. Only procedures with at least ten successful acquisitions and a success rate of at least $60 \%$ were considered reliable and recorded.

Descriptive statistical measures such as mean, standard deviation, median, range, etc for continuous variables and percentages for categorical variables had been reported to summarize the data distribution. Continuous variables between two groups were compared by independent t-test/ Mann-Whitney $U$ test wherever applicable and the three groups were compared by using one way ANOVA. The associations between two independent categorical variables were assessed through the use of Chi-square test/ Fisher's exact test wherever applicable. 
All statistical analyses were carried out with statistical software SPSS version 16.0. Results were considered statistically significant with $\mathrm{p}$ value less than 0.05 .

\section{RESULTS}

A total of 51 apparently healthy volunteers were enrolled in this study. Six of them were excluded in which two volunteers had raised transaminases, two had fatty liver in ultrasonography and two had both raised transaminases and fatty liver. Of the remaining 45 volunteer subjects with normal liver biochemistries and normal liver ultrasonography were taken for subsequent analysis. Demographic profiles and vital parameters of the study subjects are given in Table 1 and laboratory parameters in Table 2. Similarly, ultrasonographic measurements of spleen and liver and fibroscan values are presented in Table 3. Among these volunteers percentage of male was higher than female $166.66 \%$ vs $33.33 \%$ ) and percentage of resident doctors was higher than nursing staff $(80 \%$ vs $20 \%)$. The mean age of our study subjects was $30.13 \pm 4.96$ years and according to gender was comparable $131.70 \pm 3.80$ vs $27.00 \pm 5.62$ years for men and women, respectively, $\mathrm{p}=0.002)$.

\begin{tabular}{|c|c|c|c|c|}
\hline Characteristics & All subjects & Men & Women & P-value \\
\hline Number (\%) & 45 & 30 & 15 & \\
\hline Age (yrs) & $30.13 \pm 4.96$ & $31.70 \pm 3.80$ & $27.00 \pm 5.62$ & 0.002 \\
\hline \multicolumn{5}{|l|}{ Occupation: } \\
\hline Doctor (\%) & $36(80.00)$ & $30(83.30)$ & $6(16.70)$ & \\
\hline Nurse (\%) & $9(20.00)$ & $0(0.00)$ & $9(100.00)$ & \\
\hline \multicolumn{5}{|l|}{ Ethnicity: } \\
\hline Brahmins (\%) & $18(40.00)$ & $13(72.20)$ & $5(27.80)$ & \\
\hline Chhetry (\%) & $9(20.00)$ & $6(66.70)$ & $3(33.30)$ & \\
\hline Newar (\%) & $7(15.60)$ & $3(42.90)$ & $4(57.1)$ & \\
\hline Others (\%) & $11(24.40)$ & $8(72.70)$ & $3(27.30)$ & 0.529 \\
\hline SBP $(\mathrm{mmHg})$ & $114.67 \pm 8.68$ & $118.00 \pm 8.45$ & $108.00 \pm 4.14$ & $<0.001$ \\
\hline $\mathrm{DBP}(\mathrm{mmHg})$ & $74.00 \pm 6.45$ & $75.33 \pm 5.71$ & $71.33 \pm 7.19$ & 0.049 \\
\hline Pulse (per minute) & $76.91 \pm 6.38$ & $76.87 \pm 5.79$ & $77.00 \pm 7.64$ & 0.948 \\
\hline Weight (Kg) & $63.08 \pm 10.59$ & $68.38 \pm 7.48$ & $52.47 \pm 7.46$ & $<0.001$ \\
\hline West circumference $(\mathrm{cm})$ & $86.24 \pm 7.29$ & $89.67 \pm 4.41$ & $79.40 \pm 7.18$ & $<0.001$ \\
\hline BMI (Kg/m2) & $23.26 \pm 2.67$ & $23.96 \pm 2.29$ & $21.85 \pm 2.90$ & 0.021 \\
\hline
\end{tabular}

Table 2. Laboratory parameters of the study subjects.

\begin{tabular}{lllll}
\hline Characteristics & All subjects & Men & Women & -value \\
Blood Sugar R (mg/dl) & $97.47 \pm 18.46$ & $98.07 \pm 18.62$ & $96.27 \pm 18.72$ & 0.762 \\
Urea (mg/dl) & $29.84 \pm 6.75$ & $30.83 \pm 6.92$ & $27.87 \pm 6.14$ & 0.167 \\
Creatinine (mg/dl) & $1.08 \pm 0.92$ & $1.01 \pm 0.12$ & $1.21 \pm 1.61$ & $<0.001$ \\
[median, range] & {$[1.00,0.60-7.00]$} & {$[1.00,0.80-1.30]$} & {$[0.80,0.60-7.00]$} & \\
Total serum bilirubin( $\mathbf{m g} /$ & $0.91 \pm 0.33$ & $0.98 \pm 0.38$ & $0.78 \pm 0.11$ & 0.010 \\
dl) & {$[0.90,0.50-2.40]$} & {$[0.90,0.50-2.40]$} & {$[0.80,0.6-0.90]$} & \\
[Median, range] & & $0.35 \pm 0.14$ & $0.29 \pm 0.07$ & 0.1597 \\
$\begin{array}{l}\text { Direct serum bilirubin }(\mathbf{m g} / \\
\text { dl) }\end{array}$ & $0.33 \pm 0.12$ & {$[0.30,0.20-0.80]$} & {$[0.30,0.20-0.40]$} &
\end{tabular}


Basnet et al. Transient Elastographic Values of Healthy Volunteers in a Tertiary Care Hospital

$\begin{array}{|lllll|}\text { AST(u/l) } & 19.47 \pm 6.68 & 20.90 \pm 7.03 & 16.60 \pm 4.95 & 0.040 \\ \text { ALT(u/l) } & 23.16 \pm 10.95 & 26.90 \pm 11.17 & 15.67 \pm 5.31 & <0.001 \\ \text { [Median, range] } & {[20.00,7-55]} & {[25.00,7-55]} & {[14.00,9-29]} & 0.133 \\ \text { ALP(u,KA) } & 81.93 \pm 19.07 & 84.97 \pm 20.14 & 75.87 \pm 15.62 & 0.178 \\ \text { Albumin(gm/dl) } & 5.06 \pm 0.43 & 5.13 \pm 0.52 & 4.94 \pm 0.12 & <0.001 \\ \text { Total Cholesterol(mg/dl) } & 181.27 \pm 27.47 & 190.83 \pm 24.65 & 162.13 \pm 22.91 & 0.001 \\ \text { Hb(gm\%) } & 14.70 \pm 1.29 & 15.18 \pm 1.14 & 13.75 \pm 104 & 0.052 \\ \text { CBCTc(/cumm) } & 7015.56 \pm 1786.69 & 7066.67 \pm 1952.25 & 6913.33 \pm 1456.45 & 0.162 \\ \text { ESR(fl) } & 83.13 \pm 12.75 & 80.53 \pm 14.42 & 88.33 \pm 6.06 & 0.108 \\ \text { Platelets (/cumm) } & 244000 \pm 59295 & 236000 \pm 54995 & 262000 \pm 65488 \\ \text { Platelet to spleen ratio } & 2618.63 \pm 726.95 & 2495.18 \pm 719.67 & 2865.52 \pm 700.03 \\ \text { (cumm/mm) } & & & \end{array}$

\begin{tabular}{|lllll|}
\hline \multicolumn{4}{|l}{ Table 3. Ultrasonographic measurements and Fibroscan values of the study subjects. } \\
\hline Characteristics & All subjects & Men & Women & P-value \\
Liver size $(\mathbf{c m})$ & $13.16 \pm 0.88$ & $13.43 \pm 0.86$ & $12.61 \pm 0.67$ & 0.002 \\
& {$[13.35,11.58-14.94$} & $13.42,11.76-14.94]$ & {$[12.42,11.58-13.73$} & \\
[Median, Range] & & & & \\
Spleen diameter(cm) & $9.50 \pm 1.12$ & $9.65 \pm 1.15$ & $9.20 \pm 1.02$ & 0.211 \\
[Median, Range] & {$[9.44,7.32-11.52]$} & {$[9.57,7.32-11.52]$} & {$[8.88,7.82-11.08$} & 0.260 \\
Liver stiffness(kPa) & $4.24 \pm 0.70$ & $4.32 \pm 0.74$ & $4.07 \pm 0.61$ & \\
[Range] & {$[2.80-5.80]$} & {$[2.80-5.80]$} & {$[3.10-5.40]$} & \\
\hline
\end{tabular}

Mean liver stiffness value of study subjects was 4.24 $\pm 0.70 \mathrm{kPa}$. Liver stiffness value was found higher in males than in females $(4.32 \pm 0.74$ vs $4.07 \pm 0.61 \mathrm{kPa}$, respectively, $\mathrm{P}=0.26$ ) but not statistically significant. We also compared BMI and liver stiffness values but no statistically significant $(P=0.377)$. The association between liver stiffness and different group of BMI are given in Table 4. Similarly, comparison between age and liver stiffness showed positive correlation ( $r$ $=0.211)$ but not statistically significant $(P=0.164)$. Platelet $(/ \mathrm{cu} \mathrm{mm}$ ) to spleen size (bipolar diameter of spleen in millimeter) ratio was calculated and found $2618.63 \pm 726.95$. Scatter diagram between age and liver stiffness is presented in Figure 1. Similarly, scatter diagram of liver stiffness and platelets count (per cubic millimeter) in Figure 2 and spleen size $(\mathrm{cm})$ in Figure 3 are shown.

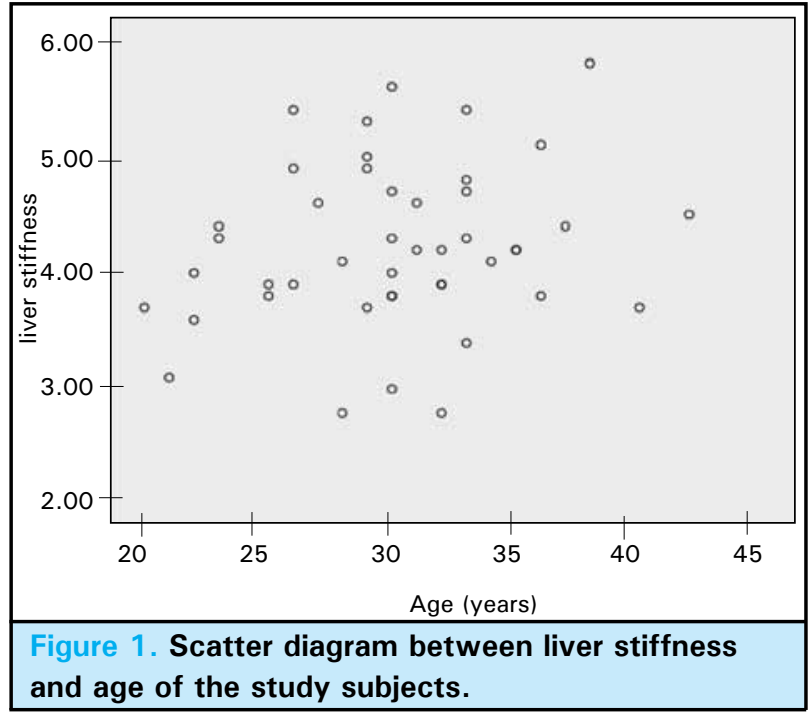




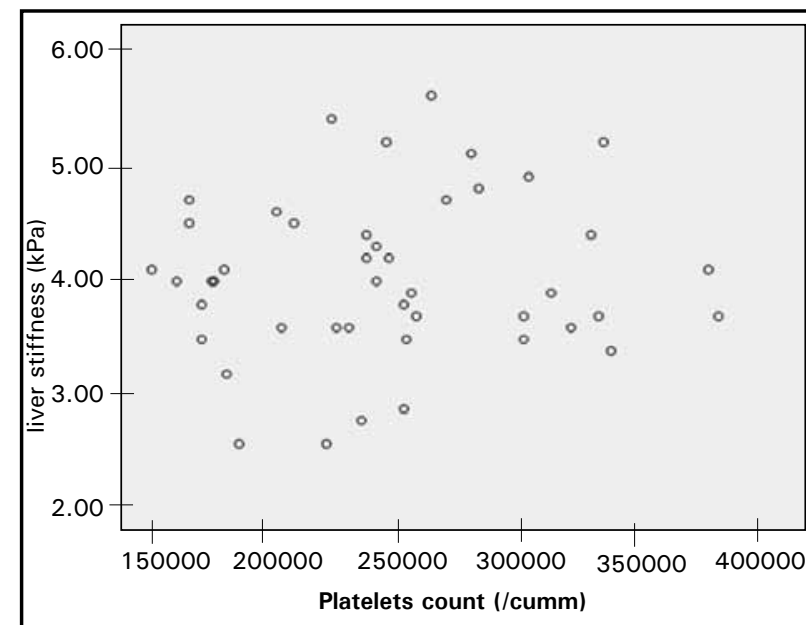

Figure 2. Scatter diagram between liver stiffness and platelet count of the study subjects.

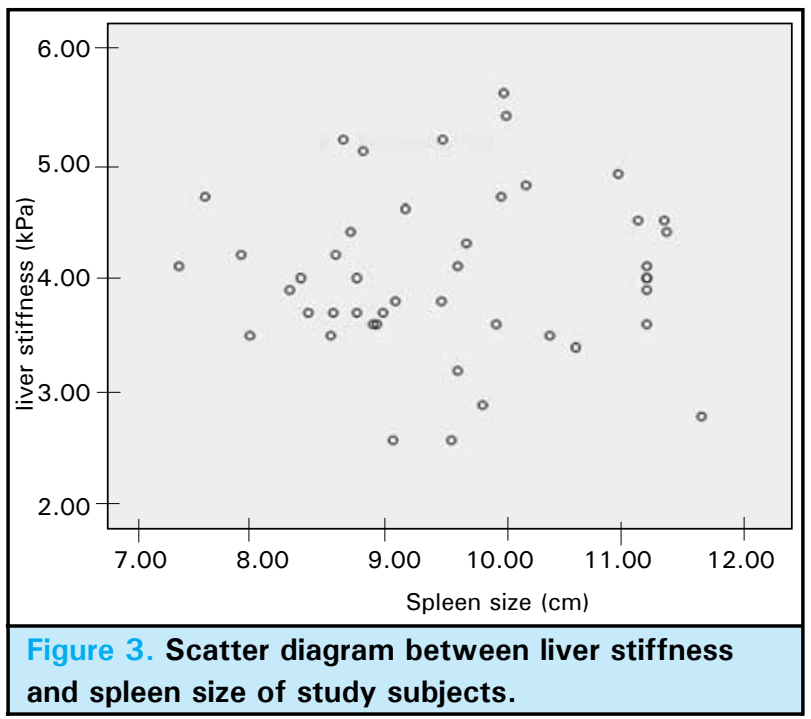

Table 4. Association between liver stiffness and different group of BMI.

\begin{tabular}{|cccc|}
\hline BMI & $\mathbf{n}$ & $\begin{array}{c}\text { Liver stiffness } \\
\text { Mean } \pm \text { S.D. }\end{array}$ & p-value \\
$18-22.9$ & 20 & $4.06 \pm 0.58$ & \\
$23-24.9$ & 14 & $4.41 \pm 1.01$ & \\
$\geq 25$ & 11 & $4.31 \pm 0.28$ & 0.3776 \\
\hline
\end{tabular}

\section{DISCUSSION}

Transient elastography (TE) is a recently described noninvasive procedure, which measures the transmission of an elastic share wave generated by vibration. This technique allows to evaluate liver stiffness. ${ }^{8}$ Briefly, TE consists of an ultrasound transducer mounted on the axis of the vibrator, which produces vibration of a mild amplitude and low frequency $(50 \mathrm{~Hz})$, consequently inducing an elastic shear wave that propagates through the liver. Pulse-echo ultrasound follows the propagation of the shear wave and measures its velocity, which is related to liver tissue stiffness. It is reported that the velocity of elastic waves is faster in fibrotic liver than normal liver. Performance of TE takes only a few minutes, and it is well tolerated by most patients. TE is performed on the right lobe of the liver with the patient lying in dorsal decubitus with the right arm in maximal abduction. Usually, 10 valid measurements are performed and median value of the ten valid measurements is considered representative of liver elasticity. Examinations with a success rate higher than $60 \%$ are considered reliable and results expressed in kilopascals (range 2.5-75 $\mathrm{kPa}$ ). The interquartile range (IQR), which represents the intrinsic variability of $T E$, $<30 \%$ of the median indicates a high-quality result. ${ }^{8}$ Transient elastography has already been validated in different liver diseases like chronic viral $C$ hepatitis, ${ }^{11-14}$ chronic viral B hepatitis, ${ }^{1516}$ HCV-HIV co- infection, ${ }^{17,18}$ liver transplant recipients, ${ }^{19-22}$ cholestatic conditions ${ }^{23}$ and hemochromatosis. ${ }^{24}$

FibroscanR is gaining popularity in various chronic liver diseases to determine status of liver in term of stiffness with different cut-off values. But data of normal values of liver stiffness is still few. We studied 45 apparently healthy volunteers with normal liver chemistries and normal liver ultrasound finding. The study subjects are few due to limitation of available resources. FibroscanR is available in single center (Center for liver diseases) and relatively inexpensive, easier to perform and safer than liver biopsy. It can also be repeated, in order to monitor changes in fibrosis during the course of treatment and can yield an immediate result after procedure. It measures liver stiffness of a value of parenchyma approximately 100 times bigger than a needle biopsy specimen so it is more representative of liver parenchyma. In this study, we found mean liver stiffness value (4.24 $\pm 0.70 \mathrm{kPa})$ in apparently healthy volunteers. Liver stiffness values were found higher in males than in females $(4.32 \pm 0.74$ vs $4.07 \pm 0.61 \mathrm{kPa}$, respectively, $\mathrm{P}=0.26$ ) but not statistically significant which is similar to Kim SU et $\mathrm{al}^{25}$ where they found the mean liver stiffness value of $4.6 \pm 0.5 \mathrm{kPa}$ (range 3.3-5.6 kPa) in their study, which was calculated from 69 strictly selected living liver and kidney donors and liver stiffness values were not significantly different between men $(4.6 \pm 0.6 \mathrm{kPa})$ and women $(4.5 \pm 0.5$ $\mathrm{kPa}, \mathrm{P}=0.636)$. Similarly, Fung et $\mathrm{al}^{26}$ reported a mean TE value in 28 healthy living-related liver donors of 4.6 (range, 2.0-7.1) kPa, and all subjects had TE values of $<7.2 \mathrm{kPa}$, which is also similar to our finding. In a study, Roulot et al $^{27}$ examined a large cohort of apparently healthy subjects with no evidence of liver diseases to establish normal liver stiffness values and mean liver stiffness was $5.49 \pm 1.59 \mathrm{kPa}$. Liver stiffness valves 
were higher in men than in women $[5.81 \pm 1.54 \mathrm{kPa}$ (range 3.8-8.0 kPa) in men vs $5.23 \pm 1.59 \mathrm{kPa}$ (range 3.3- $7.8 \mathrm{kPa}$ ) in women, $\mathrm{P}<0.002$ in contrast to our finding they found it statistically significant.

Similarly, Colombo et al, $^{28}$ found mean liver stiffness value of $4.9 \mathrm{kPa}$ ( $95 \%$ confidence interval, 4.6 - 5.1 $\mathrm{kPa}$ ) in 327 voluntary blood donors and liver stiffness values were not significantly different genders which is similar to our finding. Likewise, Sirli et al, ${ }^{29}$ in their study of 144 normal subjects in Romania found liver stiffness value of $4.8 \pm 1.3 \mathrm{kPa}$ (range $2.3-8.8 \mathrm{kPa}$ ) which is comparable to ours. But they found that liver stiffness valves of men $5.1 \pm 1.2 \mathrm{kPa}$ and women $4.6 \pm 1.2 \mathrm{kPa}$ which was significantly different in terms of gender $\mathrm{P}<$ 0.01 where our finding was not significant. Corpechot et $\mathrm{al}^{30}$ in their study of liver stiffness value in healthy men and women found that liver stiffness values were higher in men than in women $(5.2 \pm 0.7$ vs $4.5 \pm 1$ $\mathrm{kPa}, \mathrm{P}<0.01)$ which is in contrast to our finding. In our study, comparison of age and liver stiffness showed positive correlation ( $r=0.211)$ but was not statistically significant $(P=0.164)$. Roulot et al, ${ }^{25}$ found liver stiffness values tended to be higher with age but not significant statistically $(P=0.06)$ which is similar to ours and Kim SU et al, ${ }^{27}$ has similar result for age $(P=$ $0.851)$. Similarly, in this study the association between liver stiffness and different group of BMI was also not significant statistically $(P=0.377)$. Similar results were reported by Kim SU et al, ${ }^{27}$ but Roulot et al, ${ }^{25}$ who found liver stiffness values were higher in subjects with metabolic syndrome than in those without $16.5 \pm 1.64$ vs $5.33 \pm 1.51 \mathrm{kPa}, \mathrm{P}<0.0001$ ). It is well known that liver stiffness is related to the degree of hepatic fibrosis, and gradually portal hypertension would be evident, spleen diameter increases and platelet count decreases. As platelets count and spleen size are thought to reflect the clinical consequences of portal hypertension. In a study, Berzigotti $A$ et al, ${ }^{31}$ reported that patients with clinically significant portal hypertension had a higher prevalence of worse liver function (higher bilirubin, lower albumin), lower platelet count and larger spleen size as compared with patients without clinically significant portal hypertension. They reported the platelets count
$(137 \pm 64 \times 109 / 1$ in training set and $116 \pm 52$ $\times 109 / \mathrm{l}$ in validation set $)$, spleen size (13.1 \pm 3.0 $\mathrm{cm}$ in training set and $15.5 \pm 2.9$ in validation set) and platelets to spleen ratio $(1156 \pm 704$ in training set and $817 \pm 443$ in validation set) which correlate with liver stiffness values $25.2 \pm 16.6 \mathrm{kPa}$ in training set and $27.4 \pm 12.1 \mathrm{kPa}$ in validation set. They concluded these values from patients with compensated cirrhosis in contrast to our values. In our study where we found platelet count $(244000 \pm 59295 /$ cumm) and spleen size $(9.50 \pm 1.12 \mathrm{~cm})$, platelets to spleen ratio $(2618.63$ $\pm 726.67)$ and liver stiffness value $4.24 \pm 0.70 \mathrm{kPa}$ which does not correlate with it, as these values are from apparently healthy volunteers.

This study is the first of its kind in Nepalese population, so is expected to serve a benchmark for future research in this field. The major limitation of our study is its small sample size in comparison to different studies discussed here but still this gave us a baseline value of liver stiffness in our population. It is recommended that future studies are required with larger sample size and in subjects of various demographic backgrounds.

\section{CONCLUSIONS}

We examined liver stiffness value of relatively small group of healthy subjects and found mean liver stiffness value of $4.24 \pm 0.70 \mathrm{kPa}\{\operatorname{male}(4.32 \pm 0.74 \mathrm{kPa})$ and female $(4.07 \pm 0.61 \mathrm{kPa})\}$. The influence of age, gender and body mass index on liver stiffness value was not significant.

\section{ACKNOWLEDGEMENTS}

We would like to thank the center for liver disease (Bijulibazar, Kathmandu) for support by performing fibroscanR of study subjects free of cost. We would also like to thank Dr. Shankar Khanal (Statistician) as well as Mr. Prakash Kumar Nepal for support with the laboratory tests. Finally, we would like thanks to all volunteers who consented and participated in this study.

\section{REFERENCES}

1. Bugianesi E, Leone N, Vanni E, Marchesini G, Brunello F, Carucci $\mathrm{P}$, et al. Expanding the natural history of nonalcoholic steatohepatitis: from cryptogenic cirrhosis to hepatocellular carcinoma. Gastroenterology. 2002;123:134-140.

2. Hui AY, Wong VW, Chan HL, Liew CT, Chan JL, Chan FK, et al. Histological progression of non-alcoholic fatty liver disease in Chinese patients. Aliment Pharmacol Ther. 2005;21:407-413.
3. NIH Consensus Statement on Management of Hepatitis C. 2002. Hepatology. 2002;36:S3-20.

4. Bravo AA, Sheth SG, Chopra S. Liver biopsy. N Engl J Med. 2001;344:495-500.

5. Cadranel JF, Rufat P, Degos F. Practices of liver biopsy in France: results of a prospective nationwide survey. For the Group of Epidemiology of the French Association for the Study of the Liver (AFEF). Hepatology. 2000;32:477-481. 
6. Castera L, Negre I, Samii K, Buffet C. Pain experienced during percutaneous liver biopsy. Hepatology. 1999;30:1521-1530.

7. Fontana RJ, Lok AS. Noninvasive monitoring of patients with chronic hepatitis C. Hepatology. 2002;36:S57-64.

8. Sandrin L, Fourquet B, Hasquenoph JM, et al. Transient elastography: a new noninvasive method for assessment of hepatic fibrosis. Ultrasound Med Biol. 2003;29:1705-13.

9. Kettaneh A, Marcellin P, Douvin C, Poupon R, Ziol M, Beaugrand $\mathrm{M}$, de Ledinghen V. Features associated with success rate and performance of fibroscan measurements for the diagnosis of cirrhosis in HCV patients: A prospective study of 935 patients. J Hepatol. 2007;46:628-634.

10. Misra A, Chowbey P, Makkar BM, et al. Consensus Statement for Diagnosis of Obesity, Abdominal Obesity and the Metabolic Syndrome for Asian Indians and Recommendations for Physical Activity, Medical and Surgical Management. JAPI. 2009;57:163-170.

11. Ziol M, Handra-Luca A, Kettaneh A, et al. Noninvasive assessment of liver fibrosis by measurement of stiffness in patients with chronic hepatitis C. Hepatology. 2005;41:48-54.

12. Castéra L, Vergniol J, Foucher J, et al. Prospective comparison of transient elastography, Fibrotest, APRI, and liver biopsy for the assessment of fibrosis in chronic hepatitis C. Gastroenterology. 2005;128:343-350.

13. Nitta Y, Kawabe N, Hashimoto S, et al. Liver stiffness measured by transient elastography correlates with fibrosis area in liver biopsy in patients with chronic hepatitis C. Hepatol Res. 2009;39:675-684.

14. Lupşor M, Badea R, Stefănescu $H$, et al. Analysis of histopathological changes that influence liver stiffness in chronic hepatitis C. Results from a cohort of 324 patients. J Gastrointestin Liver Dis. 2008;17:155-163.

15. Chan HL, Wong GL, Choi PC, et al. Alanine aminotransferasebased algorithms of liver stiffness measurement by transient elastography (Fibroscan) for liver fibrosis in chronic hepatitis B. J Viral Hepat. 2009;16:36-44.

16. Marcellin $\mathrm{P}, \mathrm{Ziol} \mathrm{M}$, Bedossa $\mathrm{P}$, et al. Non-invasive assessment of liver fibrosis by stiffness measurement in patients with chronic hepatitis B. Liver Int. 2009;29:242-247.

17. Lédinghen V, Douvin C, Kettaneh A, et al. Diagnosis of hepatic fibrosis and cirrhosis by transient elastography in $\mathrm{HIV} /$ hepatitis C virus-coinfected patients. J Acquir Immune Defi c Syndr. 2006;41:175-179.

18. Vergara S, Macías J, Rivero A, et al. The use of transient elastometry for assessing liver fibrosis in patients with HIV and hepatitis C virus coinfection.Clin Infect Dis. 2007;45:969-974.

19. Carrion JA, Navasa M, Bosch J, Bruguera M, Gilabert R, Forns X. Transient elastography for diagnosis of advanced fibrosis and portal hypertension in patients with hepatitis $C$ recurrence after liver transplantation. Liver Transpl. 2006;12:1791-1798.
20. Rigamonti C, Donato MF, Fraquelli M, et al. Transient elastography predicts fibrosis progression in patients with recurrent hepatitis $C$ after liver transplantation. Gut. 2008;57:821-827.

21. Corradi F, Piscaglia F, Flori S, et al. Assessment of liver fibrosis in transplant recipients with recurrent $\mathrm{HCV}$ infection: usefulness of transient elastography. Dig Liver Dis. 2009;41:217-225.

22. Harada N, Soejima Y, Taketomi A, et al. Assessment of graft fibrosis by transient elastography in patients with recurrent hepatitis $\mathrm{C}$ after living donor liver transplantation. Transplantation. 2008;85:69-74.

23. Corpechot $C$, El Naggar A, Poujol-Robert A, et al. Assessment of biliary fibrosis by transient elastography in patients with PBC and PSC. Hepatology. 2006;43:1118-1124.

24. Adhoute X, Foucher J, Laharie D, et al. Diagnosis of liver fibrosis using FibroScan and other noninvasive methods in patients with hemochromatosis: a prospective study. GastroenterolClin Biol. 2008;32:180-187.

25. Kim SU, Choi GH, Han WK, Kim BK, Park JY, Kim do Y, et al. What are 'true normal' liver stiffness values using FibroScan: a prospective study in healthy living liver and kidney donors in South Korea. Liver Int. 2010;30:268-274.

26. Fung J, Lai CL, Chan SC, But D, Seto WK, Cheng C, et al. Correlation of liver stiffness and histological features in healthy persons and in patients with occult hepatitis B, chronic active hepatitis B, or hepatitis B cirrhosis. Am J Gastroenterol. 2010;105:1116-1122.

27. Roulot D, Czernichow S, Le Clésiau H, Costes JL, Vergnaud $\mathrm{AC}$, Beaugrand M. Liver stiffness values in apparently healthy subjects: influence of gender and metabolic syndrome. J Hepatol. 2008;48:606-613.

28. Colombo S, Belloli L, Buonocore M, et al. Liver stiffness values in the normal population: a studying voluntaryblood donors. Hepatology. 2008;48(Suppl):A995.

29. Sirli R, Sporea I, Tudora A, et al. Transient elastographic evaluation of subjects without known hepatic pathology: does age change the liver stiffness? J Gastrointestin Liver Dis. 2009; 18:57-60.

30. Corpechot C, El Naggar A, Poupon R. Gender and liver: is the liver stiffness weaker in weaker sex? Hepatology. 2006;44:513-4.

31. Berzigotti A, Seijo S, Arena U, Abrajdes JG, Vizzutti F,Garcia-Pagan JC ,Pinzani M, Bosch J. Elastography, spleen size, and platelet count identify portal hypertension in patients with compensated cirrhosis.Gastroenterology,2013 ;144:102-111. 